\title{
Estimating glomerular filtration rates in elderly Chinese patients with chronic kidney disease: performance of six modified formulae developed in Asian populations
}

\author{
This article was published in the following Dove Press journal: \\ Clinical Interventions in Aging \\ 16 July 2013 \\ Number of times this article has been viewed
}

\begin{abstract}
Xun $\operatorname{Liu}^{1,2, *}$
Haixia $X^{3, *}$

Zebin Zheng ${ }^{4,5, *}$

Cheng Wang'

Cailian Cheng'

Chenggang Shi ${ }^{1}$

Yihong Gong ${ }^{4,5}$

Ming $\mathrm{Li}^{\prime}$

Tanqi Lou'

'Division of Nephrology, Department of Internal Medicine, The Third

Affiliated Hospital of Sun Yat-sen

University, ${ }^{2}$ College of Biology

Engineering, South China

University of Technology, ${ }^{3}$ Division

of Endocrinology, Department

of Internal Medicine, The Third

Affiliated Hospital of Sun Yat-sen

University, Guangzhou, People's

Republic of China; ${ }^{4}$ Department of

Biomedical Engineering, School of

Engineering, Sun Yat-sen University,

Guangzhou, Guangdong, People's

Republic of China; ${ }^{5}$ Guangdong

Provincial Key Laboratory of

Sensor Technology and Biomedical

Instruments, Sun Yat-sen University,

Guangzhou, Guangdong, People's

Republic of China

*These authors contributed equally to this work
\end{abstract}

Correspondence: Tanqi Lou

Division of Nephrology, Department

of Internal Medicine, The Third

Affiliated Hospital of Sun Yat-sen

University, Guangzhou, 510630 People's

Republic of China

Email lou.tq@163.com

\begin{abstract}
Objectives: The aim of the present study was to evaluate modified glomerular filtration rate (GFR) prediction formulae in an elderly Chinese population with chronic kidney disease (CKD).

Methods: A total of 378 elderly Chinese patients with CKD were enrolled. The GFR was estimated with six modified GFR prediction formulae. The performances of the estimated GFRs were compared with those of the standard GFRs measured by technetium-99m diethylenetraminepentaacetic acid.

Results: Biases were similar for Chinese formula 1, the Asian formula, and Chinese formula 2 (median difference, $2.22 \mathrm{~mL} / \mathrm{min} / 1.73 \mathrm{~m}^{2}$ and $2.59 \mathrm{~mL} / \mathrm{min} / 1.73 \mathrm{~m}^{2}$ for Chinese formula 1 and the Asian formula, respectively, versus (vs) $3.69 \mathrm{~mL} / \mathrm{min} / 1.73 \mathrm{~m}^{2}$ for Chinese formula $2[P=0.298$ and $P=0.913$, respectively]). Precision was improved with the Japanese formula (interquartile range of the difference, $3.14 \mathrm{~mL} / \mathrm{min} / 1.73 \mathrm{~m}^{2}$ of the Japanese formula versus $15.53-23.06 \mathrm{~mL} /$ $\mathrm{min} / 1.73 \mathrm{~m}^{2}$ of the other formulae). The accuracy of Chinese formula 2 was the highest $(30 \%$ accuracy, $59.3 \%$ vs range $37.8-54.0 \%$ [ $P<0.05$ for all comparisons $])$. However, none of the modified formulae surpassed the acceptable tolerance $(>70 \%)$, and the GFR category misclassification rates for all the formulae exceeded $50 \%$.

Conclusion: Our findings suggest that all six modified formulae developed in Asian populations may show great bias in elderly Chinese patients with CKD. Also, our study suggests the need for uniform measures for the assessment of CKD in the elderly to guarantee better sensitivity and specificity.
\end{abstract}

Keywords: formula, CKD, Asian, GFR

\section{Introduction}

Accurate estimation of glomerular filtration rate (GFR) is essential for the detection, diagnosis, and management of patients with chronic kidney disease (CKD). ${ }^{1}$ The incidence of CKD is markedly high in elderly populations. ${ }^{2}$ The Modification of Diet in Renal Disease (MDRD) formula and the Chronic Kidney Disease Epidemiology Collaboration (CKD-EPI) formula are the most frequently used formulae for estimating GFR but are known to be less accurate for racial and ethnic groups outside North America, Europe, and Australia. ${ }^{3}$ Taking this into account, six GFR prediction formulae, the Asian formula, ${ }^{4}$ Korean formula, ${ }^{5}$ Japanese formula, ${ }^{6}$ Thai formula, ${ }^{7}$ Chinese formula $1,{ }^{8}$ and Chinese formula $2,{ }^{9}$ were developed based on Asian populations either by adding a coefficient into the original MDRD formula or developing a new equation using the same variables as the MDRD formula. These modified formulae 
have not been validated in elderly CKD patients. The current study was designed to compare the performance of different modified formulae for the estimation of GFR in elderly Chinese patients with CKD.

\section{Materials and methods}

\section{Sample size calculation}

Based on the findings of a pilot study (Tables S1 and S2), the minimum sample size was calculated in accordance with the formula $^{10}$ for a cross-sectional survey. A significance level of $95 \%$ and $80 \%$ power was used. We therefore estimated that the sample size should be 360 subjects.

\section{Study design}

A cross-sectional, single-center study design was used. The study, approved by the institutional review board at the Third Affiliated Hospital of Sun Yat-sen University, was conducted in accordance with the ethical guidelines of the Helsinki Declaration. Informed consent was obtained from each patient before the study.

\section{Subjects}

A total of 378 elderly CKD patients were referred consecutively to our department in the Third Affiliated Hospital of Sun Yat-sen University, People's Republic of China, for measurement of their GFR from January 2005 through December 2010. The mean age of the patients was $72.8 \pm 5.7$ (range 65-93) years, with standard glomerular filtration rate (sGFR) measured by technetium-99m diethylenetriaminepentaacetic acid ( ${ }^{99 \mathrm{~m}}$ Tc-DTPA) renal scintigraphic analysis found to be $39.5 \pm 20.2$ (range $4.5-95.1$ ) $\mathrm{mL} / \mathrm{min} / 1.73 \mathrm{~m}^{2}$ (Table 1). Inclusion criteria were age 65 years or older and CKD diagnosed and categorized according to the Kidney Disease: Improving Global Outcomes clinical practice guidelines. ${ }^{11}$ Exclusion criteria are described elsewhere. ${ }^{12}$

\section{Measurements}

The sGFR was determined by ${ }^{99 \mathrm{~m}}$ Tc-DTPA renal scintigraphic analysis, ${ }^{13}$ measured using a commercial SPECT/CT system (Discovery VH, GE Healthcare, Waukesha, WI, USA). A high correlation was observed in comparison to renal scintigraphic analysis with inulin clearance, the reference standard of GFR measurement. ${ }^{14}$ Serum creatinine (SC) was analyzed using the enzymatic method on a Hitachi 7180 analyzer (Tokyo, Japan; reagents from Roche Diagnostics, Mannheim, Germany), traceable to isotope dilution mass spectrometry.

We used six modified formulae to calculate the estimated glomerular filtration rate (eGFR):
Table I Patient characteristics*

\begin{tabular}{ll}
\hline Subjects $(\mathrm{n})$ & 378 \\
Age (years) & $72.8 \pm 5.7$ \\
Male sex $(\mathrm{n}, \%)$ & $242(64.0)$ \\
Diabetes $(\mathrm{n}, \%)$ & $162(42.8)$ \\
Body mass index & \\
$\quad$ Mean $\left(\mathrm{kg} / \mathrm{m}^{2}\right)$ & $23.3 \pm 3.6$ \\
$<20(\mathrm{n}, \%)$ & $67(17.7)$ \\
$20-24(\mathrm{n}, \%)$ & $196(51.9)$ \\
$25-30(\mathrm{n}, \%)$ & $97(25.7)$ \\
$>30(\mathrm{n}, \%)$ & $18(4.8)$ \\
Weight $(\mathrm{kg})$ & $61.3 \pm 11.5$ \\
Height $(\mathrm{cm})$ & $161.7 \pm 8.1$ \\
Body-surface area $\left(\mathrm{m}^{2}\right)$ & $1.64 \pm 0.17$ \\
Serum creatinine $(\mathrm{mg} / \mathrm{dL})$ & $2.5 \pm 1.9$ \\
Measured GFR $\left(\mathrm{mL} / \mathrm{min} / \mathrm{l} .73 \mathrm{~m}^{2}\right)$ & \\
Mean & $39.5 \pm 20.2$ \\
$<15$ & $43(\mathrm{II} .4)$ \\
I5-29 & $94(24.9)$ \\
$30-44$ & $96(25.4)$ \\
$45-59$ & $87(23.0)$ \\
$60-89$ & $53(14.0)$ \\
$>90$ & $5(1.3)$ \\
\hline
\end{tabular}

Note: *Quantitative data are expressed as mean \pm standard deviation; categorical data, as number (percentage).

Abbreviation: GFR, glomerular filtration rate.

- The Asian formula: ${ }^{4}$ $\mathrm{GFR}=1.086 \times 175 \times \mathrm{SC}^{-1.154} \times \mathrm{age}^{-0.203} \times[0.742$ if patient is female]

- The Korean formula: ${ }^{5}$

$$
\begin{aligned}
& \mathrm{GFR}= 87.832 \times \mathrm{SC}^{-0.882} \times \mathrm{age}^{0.01} \times[0.653 \text { if patient is } \\
&\text { female }]
\end{aligned}
$$

- The Japanese formula: ${ }^{6}$

GFR $=194 \times \mathrm{SC}^{-1.094} \times$ age $^{-0.287} \times[0.739$ if patient is female]

- The Thai formula: ${ }^{7}$

$\mathrm{GFR}=1.129 \times 175 \times \mathrm{SC}^{-1.154} \times \mathrm{age}^{-0.203} \times[0.742$ if patient is female]

- Chinese formula 1:8

$\mathrm{GFR}=175 \times \mathrm{SC}^{-1.234} \times \mathrm{age}^{-0.179} \times[0.79$ if patient is female]

- Chinese formula $2:{ }^{9}$

$\mathrm{GFR}=234.96 \times \mathrm{SC}^{-0.926} \times \mathrm{age}^{-0.280} \times[0.828$ if patient is female].

\section{Statistical analysis}

The difference between the eGFR and sGFR was defined as eGFR minus sGFR. Accuracy was measured as the percentage of eGFR not deviating more than $30 \%$ from the sGFR.

A Wilcoxon Mann-Whitney test was used for the difference, bootstrap method for IQR for difference, and $\chi^{2}$ test for accuracy, respectively. In a pilot study (Tables S1 
and S2), Chinese formula 2 performed better than the other formulae. Therefore, we chose eGFR measured by Chinese formula 2 as the reference against which all comparisons among formulae were made. Performances of the modified formulae were assessed in terms of four factors - bias, precision, accuracy, and GFR category misclassification rate. An optimal score system was developed. The formula that performed the best in each aspect in the entire population was scored as 1, and in each GFR subgroup the best scores were 0.5 . The greater the total scores, the better the synthetic performance. All statistical analyses were performed using SPSS (v 11.0; IBM Corporation, Armonk, NY, USA) and Matlab (v 2011b; The MathWorks ${ }^{\circledR}$, Natick, MA, USA) software.

\section{Results}

Biases were similar for Chinese formula 1, the Asian formula, and Chinese formula 2 (median difference, $2.22 \mathrm{~mL} / \mathrm{min} / 1.73 \mathrm{~m}^{2}$ and $2.59 \mathrm{~mL} / \mathrm{min} / 1.73 \mathrm{~m}^{2}$ for Chinese formula 1 and the Asian formula, respectively, vs $3.69 \mathrm{~mL} / \mathrm{min} / 1.73 \mathrm{~m}^{2}$ for Chinese formula $2[P=0.298$ and $P=0.913$, respectively]), while bias for the Chinese equation 2 was less than those for the Korean formula, Japanese formula, and Thai formula (median difference, range -6.71 to $11.72 \mathrm{~mL} / \mathrm{min} / 1.73 \mathrm{~m}^{2}$ $[P<0.001$ for all comparisons $]$ ). Precision was improved with the Japanese formula (IQR of the difference, $13.14 \mathrm{~mL} / \mathrm{min} /$ $1.73 \mathrm{~m}^{2}$ of the Japanese formula versus $15.53-23.06 \mathrm{~mL} / \mathrm{min} /$ $1.73 \mathrm{~m}^{2}$ of the other formulae). The accuracy of Chinese formula 2 was the highest (30\% accuracy, 59.3 vs range $37.8 \%-54.0 \%,[P<0.05$ for all comparisons $])$. There was also an improvement in the GFR category misclassification rate of Chinese formula 2 (54.0 vs range $57.4 \%-68.3 \%[P<0.001$ for the Asian formula, Korean formula, Thai formula and Chinese formula $1 ; P=0.320$ for the Japanese formula]). However, none of the modified formulae surpassed the acceptable tolerance $(>70 \%)$, and the GFR category misclassification rate of all the formulae exceeded $50 \%$ (Table 2).

The performances of the six modified formulae in various GFR categories were analyzed. In the subgroup with sGFR $<30 \mathrm{~mL} / \mathrm{min} / 1.73 \mathrm{~m}^{2}$, the bias of Chinese formula 2 was more than those of the Thai formula and Asian formula (median difference, $P<0.001$ for both comparisons), while it was less than those of Chinese formula 1, the Japanese formula, and the Korean formula (median difference, $P<0.001$ for all comparisons). Chinese formula 2 showed the lowest GFR category misclassification rate $(P<0.01$ for all comparisons except the Japanese formula, for which $P=0.272$ ). Among all three subgroups, precision was improved with the
Table 2 Performance between measured glomerular filtration rate (GFR) and estimated GFR

\begin{tabular}{|c|c|c|c|c|}
\hline \multirow[t]{2}{*}{ Variable } & \multicolumn{4}{|c|}{ Measured GFR $\left(\mathrm{mL} / \mathrm{min} / \mathrm{l} .73 \mathrm{~m}^{2}\right)$} \\
\hline & Overall & $<\mathbf{3 0}$ & $30-59$ & $\geq 60$ \\
\hline \multicolumn{5}{|c|}{ Bias, median difference $\left(\mathrm{mL} / \mathrm{min} / \mathrm{l} .73 \mathrm{~m}^{2}\right)$} \\
\hline Asian formula & 2.59 & $-1.48 *$ & $5.3 \mathrm{I}^{\dagger}$ & $10.79 *$ \\
\hline Korean formula & $11.72 *$ & $7.17^{*}$ & $17.25 *$ & $14.85 *$ \\
\hline Japanese formula & $-6.71 *$ & $-4.94 *$ & $-7.49 *$ & $-12.38^{*}$ \\
\hline Thai formula & $3.74 *$ & $-0.98 *$ & $7.43 *$ & $14.47 *$ \\
\hline Chinese formula I & 2.22 & $-2.66 *$ & $5.58 *$ & $18.47^{*}$ \\
\hline Chinese formula 2 & 3.69 & 1.80 & 4.85 & 4.38 \\
\hline \multicolumn{5}{|c|}{ Precision, IQR of the difference $\left(\mathrm{mL} / \mathrm{min} / \mathrm{I.73} \mathrm{m}^{2}\right)$} \\
\hline Asian formula & $21.40^{*}$ & $10.96 *$ & $23.28 *$ & $36.10^{*}$ \\
\hline Korean formula & $22.06 *$ & $13.90 *$ & $25.52 *$ & $30.08 *$ \\
\hline Japanese formula & $13.14 *$ & $9.77 *$ & $14.48^{*}$ & $25.61 *$ \\
\hline Thai formula & $22.48^{*}$ & $11.25 *$ & $24.07^{*}$ & $37.7 I^{*}$ \\
\hline Chinese formula I & $23.06 *$ & $10.62^{\dagger}$ & $24.98 *$ & $42.59 *$ \\
\hline Chinese formula 2 & 15.53 & 10.68 & 17.70 & 28.05 \\
\hline \multicolumn{5}{|c|}{ Accuracy, $\mathbf{3 0} \%$ accuracy (\%) } \\
\hline Asian formula & $49.2 *$ & $45.3^{*}$ & $53.0 *$ & $46.6^{*}$ \\
\hline Korean formula & $37.8^{*}$ & $33.6^{*}$ & $37.2^{*}$ & $50.0^{\dagger}$ \\
\hline Japanese formula & $54.0^{\ddagger}$ & $42.3^{\dagger}$ & $58.5^{\ddagger}$ & $67.2^{\ddagger}$ \\
\hline Thai formula & $48.4^{*}$ & $46.0 *$ & $50.3^{*}$ & $48.3^{*}$ \\
\hline Chinese formula I & $47.4^{*}$ & $44.5^{*}$ & $50.3^{*}$ & $44.8^{\dagger}$ \\
\hline Chinese formula 2 & 59.3 & 48.9 & 63.9 & 69.0 \\
\hline \multicolumn{5}{|c|}{ GFR category misclassification rate (\%) } \\
\hline Asian formula & $57.4^{*}$ & $43.8^{*}$ & $68.3^{*}$ & $55.2^{*}$ \\
\hline Korean formula & $68.3^{*}$ & $57.7^{*}$ & $80.3^{\dagger}$ & $55.2^{\dagger}$ \\
\hline Japanese formula & 58.5 & 48.2 & $65.0^{\dagger}$ & $62.1^{\dagger}$ \\
\hline Thai formula & $59.5^{*}$ & $43.8^{*}$ & $72.1 *$ & $56.9 *$ \\
\hline Chinese formula I & $58.7^{*}$ & $41.6^{\dagger}$ & $70.5^{*}$ & $62.1^{*}$ \\
\hline Chinese formula 2 & 54.0 & 43.8 & 63.4 & 48.3 \\
\hline
\end{tabular}

Notes: $* P<0.001$ compared with Chinese formula 2-GFR; $+P<0.01$ compared with Chinese formula 2-GFR; ${ }^{*}<0.05$ compared with Chinese formula 2-GFR. Abbreviations: GFR, glomerular filtration rate; IQR, interquartile range.

Japanese formula (IQR, $P<0.001$ for all comparisons), and accuracy with Chinese formula 2 (30\% accuracy, $P<0.05$ for all comparisons). In the subgroups with sGFR $30-59 \mathrm{~mL} / \mathrm{min} / 1.73 \mathrm{~m}^{2}$ as well as $>60 \mathrm{~mL} / \mathrm{min} / 1.73 \mathrm{~m}^{2}$, there were improvements in both the bias and GFR category misclassification rate of Chinese formula $2(P<0.01$ for all comparisons). Detailed performances are listed in Table 2.

An optimal score system was developed to synthetically evaluate the performances of different modified formulae (Table 3). Chinese formula 2 achieved the greatest total scores (5.5 vs range $0.0-2.5$ for the rest of the formulae).

\section{Discussion}

In this study, for the first time as far as we are aware, six modified formulae derived from Asian populations were validated for the estimation of GFR in elderly Chinese patients with CKD. We found that none of the formulae had 30\% accuracy up to the acceptable tolerance $(>70 \%)$, and the GFR category 
Table 3 Optimal scores* by different formulae

\begin{tabular}{ll}
\hline Formula & Total score \\
\hline Asian & 0.0 \\
Korean & 0.0 \\
Japanese & 2.5 \\
Thai & 0.5 \\
Chinese I & 1.5 \\
Chinese 2 & 5.5
\end{tabular}

Note: $*$ The formula that performs the best in each aspect in the entire population scored I and in each GFR subgroup scored 0.5.

misclassification rate of all formulae exceeded 50\%. Such results are consistent with previous findings ${ }^{15}$ and suggest that other factors in addition to race or ethnicity may affect the performance of GFR prediction formulae.

Study population was the first of these suggested factors. All the currently available modified formulae were derived from the general Asian population. However, the validation population in this study was elderly CKD patients. Intrinsic factors, such as the loss of muscle mass with aging, ${ }^{16}$ affect the evaluation of GFR in elderly CKD patients. Other studies have reported mean sGFRs ranging from 50.7 to $59.1 \mathrm{~mL} / \mathrm{min} / 1.73 \mathrm{~m}^{2},{ }^{4-9}$ which is much higher than that of the validation population in this study. The distribution of the GFR categories in our study was also different from the other studies. Differences in the study population characteristics between the original development dataset and the validation dataset led to bias in the modified GFRestimating formulae. ${ }^{17}$

The second factor is the method used to measure GFR. Renal inulin clearance was used as the sGFR in the Korean formula ${ }^{5}$ and Japanese formula,${ }^{6}$ which was different from the plasma clearance of diethylenetriaminepentaacetic acid (DTPA) used for the Asian formula, ${ }^{4}$ Thai formula, ${ }^{7}$ and Chinese formula $1,{ }^{8}$ and the DTPA renal dynamic imaging in both Chinese formula $2^{9}$ and in this study as well. Rehling et a ${ }^{18}$ found that renal dynamic imaging gave more accurate values than the renal clearance of inulin, whereas Zuo et $\mathrm{al}^{19}$ indicated that the plasma clearance of DTPA was systemically higher than that of modified inulin. Variability in the measurement of GFR introduces error into the estimation of GFR. ${ }^{20}$

Calibration of SC assays was the third factor. The SC value was measured by the enzyme method calibrated to the Cleveland Clinic Laboratory for both the Japanese formula ${ }^{6}$ and Chinese formula $1 .{ }^{8}$ The SC value in Chinese formula $2^{9}$ was obtained by Jaffe's kinetic method. SC levels in the Asian formula, ${ }^{4}$ Korean formula, ${ }^{5}$ Thai formula, ${ }^{7}$ and this study were all calibrated to an assay traceable to isotope dilution mass spectrometry. The inaccuracy in the modified formulae may be due in part to the differences in the calibration of $\mathrm{SC}$ assays. ${ }^{21}$

\section{Conclusion}

Our findings suggest that all six modified formulae developed in Asian populations may show great bias in elderly Chinese patients with CKD. Our study also suggests the need for uniform measures for the assessment of CKD in the elderly to guarantee better sensitivity and specificity. Further studies should compare different GFR-estimating formulae in similar population cohorts with the same GFR measurement and SC calibration.

\section{Acknowledgments}

We would like to thank all the doctors, nurses, technicians, and patients involved in this study for their cooperation. We would also like to thank Dr David Cushley from International Science Editing, Clare, Ireland, for assistance with the English version of the article.

This work was supported by the National Natural Science Foundation of China (Grant No. 81070612). This work was also supported by the China Postdoctoral Science Foundation (Grant No 201104335), Guangdong Science and Technology Plan (Grant No 2011B031800084), the Fundamental Research Funds for the Central Universities (Grant No 11ykpy38), and the National Project of Scientific and Technical Supporting Programs Funded by the Ministry of Science and Technology of China (Grant No 2011BAI10B05).

\section{Disclosure}

The authors declare no conflicts of interest in this work.

\section{References}

1. Levey AS, Coresh J. Chronic kidney disease. Lancet. 2012;379(9811): $165-180$.

2. Phoon RK. Chronic kidney disease in the elderly - assessment and management. Aust Fam Physician. 2012;41(12):940-944.

3. Earley A, Miskulin D, Lamb EJ, Levey AS, Uhlig K. Estimating equations for glomerular filtration rate in the era of creatinine standardization: a systematic review. Ann Intern Med. 2012;156(11):785-795, W-270, W-271, W-272, W-273, W-274, W-275, W-276, W-277, W-278.

4. Teo BW, Xu H, Wang D, et al. GFR estimating equations in a multiethnic Asian population. Am J Kidney Dis. 2011;58(1):56-63.

5. Lee CS, Cha RH, Lim YH, et al. Ethnic coefficients for glomerular filtration rate estimation by the Modification of Diet in Renal Disease study equations in the Korean population. J Korean Med Sci. 2010;25(11): $1616-1625$.

6. Matsuo S, Imai E, Horio M, et al; Collaborators developing the Japanese equation for estimated GFR. Revised equations for estimating glomerular filtration rate (GFR) from serum creatinine in Japan. Am J Kidney Dis. 2009;53(6):982-992. 
7. Praditpornsilpa K, Townamchai N, Chaiwatanarat T, et al. The need for robust validation for MDRD-based glomerular filtration rate estimation in various CKD populations. Nephrol Dial Transplant. 2011;26(9): 2780-2785.

8. Ma YC, Zuo L, Chen JH, et al. Modified glomerular filtration rate estimating equation for Chinese patients with chronic kidney disease. J Am Soc Nephrol. 2006;17(10):2937-2944.

9. Shi H, Chen N, Zhang W, et al. Evaluating and refitting the simplified equation of MDRD to predict glomerular filtration rate in Chinese patients with chronic kidney disease. Chin J Pract Int Med. 2006;26(9): 665-669.

10. Jones SR, Carley S, Harrison M. An introduction to power and sample size estimation. Emerg Med J. 2003;20(5):453-458

11. Levey AS, de Jong PE, Coresh J, et al. The definition, classification, and prognosis of chronic kidney disease: a KDIGO Controversies Conference report. Kidney Int. 2011;80(1):17-28.

12. Xun L, Cheng W, Hua T, et al. Assessing glomerular filtration rate (GFR) in elderly Chinese patients with chronic kidney disease (CKD): a comparison of various predictive equations. Arch Gerontol Geriatr. 2010;51(1):13-20.

13. Liu X, Lv L, Wang C, et al. Comparison of prediction equations to estimate glomerular filtration rate in Chinese patients with chronic kidney disease. Intern Med J. 2012;42(5):e59-e67.

14. Chen LI, Kuo MC, Hwang SJ, Chen YW, Wu KD, Chen HC. Comparisons of technetium-99m diethylenetriaminepentaacetic acid plasma clearance and renal dynamic imaging with inulin clearance. Am J Kidney Dis. 2011;58(6):1043-1045.
15. Rule AD, Teo BW. GFR estimation in Japan and China: what accounts for the difference? Am J Kidney Dis. 2009;53(6):932-935.

16. Fielding RA, Vellas B, Evans WJ, et al. Sarcopenia: an undiagnosed condition in older adults. Current consensus definition: prevalence, etiology, and consequences. International working group on sarcopenia. J Am Med Dir Assoc. 2011;12(4):249-256.

17. Ma YC, Zuo L, Su ZM, et al. Distribution of reference GFR in a development population: a critical factor for the establishment of a GFR estimation equation. Clin Nephrol. 2011;76(4):296-305.

18. Rehling M, Møller ML, Thamdrup B, Lund JO, Trap-Jensen J. Reliability of a 99mTc-DTPA gamma camera technique for determination of single kidney glomerular filtration rate. A comparison to plasma clearance of $51 \mathrm{Cr}$-EDTA in one-kidney patients, using the renal clearance of inulin as a reference. Scand J Urol Nephrol. 1986;20(1):57-62.

19. Zuo L, Dai SS. The discrepancy between two reference GFR measurements: plasma clearance of ${ }^{99 \mathrm{~m} T C-D T P A}$ used in China and a modified renal clearance of insulin used in Japan. J Am Soc Nephrol. 2010;21(Suppl):177A.

20. Kwong YT, Stevens LA, Selvin E, et al. Imprecision of urinary iothalamate clearance as a gold-standard measure of GFR decreases the diagnostic accuracy of kidney function estimating equations. $\mathrm{Am} \mathrm{J}$ Kidney Dis. 2010;56(1):39-49.

21. Vickery S, Stevens PE, Dalton RN, van Lente F, Lamb EJ. Does the ID-MS traceable MDRD equation work and is it suitable for use with compensated Jaffe and enzymatic creatinine assays? Nephrol Dial Transplant. 2006;21(9):2439-2445. 


\section{Supplementary tables}

Table SI Clinical characteristics* of a subgroup of patients selected from January 2005 to December 2008

\begin{tabular}{ll}
\hline Subjects $(\mathrm{n})$ & 215 \\
Age (years) & $72.9 \pm 5.9$ \\
Male sex $(\mathrm{n}, \%)$ & $130(60.5)$ \\
Diabetes $(\mathrm{n}, \%)$ & $95(44.1)$ \\
Body mass index & \\
$\quad$ Mean $\left(\mathrm{kg} / \mathrm{m}^{2}\right)$ & $23.7 \pm 3.7$ \\
$\quad<20(\mathrm{n}, \%)$ & $32(14.9)$ \\
$20-24(\mathrm{n}, \%)$ & $110(51.2)$ \\
$25-30(\mathrm{n}, \%)$ & $58(27.0)$ \\
$>30(\mathrm{n}, \%)$ & $15(7.0)$ \\
Weight $(\mathrm{kg})$ & $61.9 \pm 12.1$ \\
Height $(\mathrm{cm})$ & $161.3 \pm 8.7$ \\
Body-surface area $\left(\mathrm{m}^{2}\right)$ & $1.64 \pm 0.18$ \\
Serum creatinine $(\mathrm{mg} / \mathrm{dL})$ & $2.4 \pm 2.0$ \\
Measured GFR $\left(\mathrm{mL} / \mathrm{min} / \mathrm{I} .73 \mathrm{~m}^{2}\right)$ & \\
Mean & $39.4 \pm 20.2$ \\
$<15$ & $4(1.9)$ \\
I5-29 & $24(I 1.2)$ \\
$30-44$ & $56(26.0)$ \\
$45-59$ & $53(24.7)$ \\
$60-68$ & $53(24.7)$ \\
$>90$ & $25(I 1.6)$ \\
\hline
\end{tabular}

Note: *Plus-minus values are means \pm standard deviation.

Abbreviation: GFR, glomerular filtration rate.
Table S2 Performance between the estimated glomerular filtration rate (GFR) and standard GFR in a subgroup of patients selected from January 2005 to December 2008

\begin{tabular}{lllll}
\hline Equation & $\begin{array}{l}\text { Median of } \\
\text { difference } \\
(\mathbf{m L} / \mathbf{m i n} /\end{array}$ & $\begin{array}{l}\text { IQR of the } \\
\text { difference } \\
(\mathbf{m L} / \mathbf{m i n} /\end{array}$ & $\begin{array}{l}\mathbf{3 0 \%} \\
\text { accuracy } \\
\mathbf{( \% )}\end{array}$ & $\begin{array}{l}\text { GFR category } \\
\text { misclassification } \\
\text { rate (\%) }\end{array}$ \\
\hline Asian & 2.61 & 21.55 & 47.0 & 58.1 \\
Korean & II.30 & 22.41 & 37.7 & 67.9 \\
Japanese & -6.30 & 13.88 & 52.6 & 54.9 \\
Thai & 3.72 & 22.88 & 46.0 & 60.9 \\
Chinese I & 2.18 & 24.30 & 46.5 & 58.6 \\
Chinese 2 & 4.00 & 16.40 & 57.7 & 56.3
\end{tabular}

Abbreviation: IQR, interquartile range.

Notes: Chinese I formula was developed by $\mathrm{Ma}$ et $\mathrm{al},{ }^{8}$ Chinese 2 formula was developed by Shi et al. ${ }^{9}$

\section{Publish your work in this journal}

Clinical Interventions in Aging is an international, peer-reviewed journal focusing on evidence-based reports on the value or lack thereof of treatments intended to prevent or delay the onset of maladaptive correlates of aging in human beings. This journal is indexed on PubMed Central, MedLine, the American Chemical Society's 'Chemical Abstracts
Service' (CAS), Scopus and the Elsevier Bibliographic databases. The manuscript management system is completely online and includes a very quick and fair peer-review system, which is all easy to use. Visit http://www.dovepress.com/testimonials.php to read real quotes from published authors. 